\title{
Application Electrical Engineering Training and Intelligent Technology of Electrical and Electronic Technology under Artificial Intelligence Technology
}

\author{
Hai $\mathrm{Pu}^{1}$ \\ ${ }^{1}$ Chongqing Industry \& Trade Polytechnic, Chongqing, China, 408000
}

\begin{abstract}
Absrtact. With the development of the times and the improvement of modern industrial technology, computer technology has been greatly developed, so a new concept has been put forward, that is, artificial intelligence. And the composition of modern life is mainly electricity, so in the current era, electronic technology has been rapidly developed. But the original electrical and electronic technology can no longer match today's intelligent technology, but electronic technology is the basis of the development of modern intelligent technology. Therefore, the purpose of this paper is to use artificial intelligence technology to study the application of electrical engineering training and intelligent technology of electrical and electronic technology. After consulting the history of electrical engineering and the development, current situation and future development direction of electrical and electronic technology, this paper reviews the algorithms constructed by artificial intelligence and the basic operation of intelligent things. The improved adaptive parameter DBSCAN clustering algorithm is used to train electrical engineering and to make reference for the intelligence of electrical and electronic technology. The experimental results show that a good algorithm can speed up the training degree of electrical engineering and speed up the intelligent progress of electrical and electronic technology.
\end{abstract}

\section{Introduction}

Ever since the discovery of electricity, the history of modern human development has made a huge leap forward. The speed of development is the fastest period in human history. Ac and the derivation of various formula theorems greatly increase the development of modern life [1]. Then with the advent of computer in 1946, the development of modern life is getting faster and faster. Human beings have machines that replace the original mode of work, that is, computers for computing and processing [2]. Therefore, in modern life, computer technology and electronic technology are two basic components, and electronic technology and information technology are complementary [3]. However, because of the rapid development of information technology in recent years, the development of electronic technology can not fully adapt to the development of information technology. Therefore, we should reform electronic information engineering and electrical and electronic technology to enable them to adapt to the development of modern electronic technology, but this is difficult. Although information technology has been available for only a few decades, these decades have made great changes people's material life [4] .

From the initial software build to the later slightly more complex programs, to the present information collection, the data size also ranges from a few bytes to dozens of bytes to the next million bytes and to the present tens of billions of bytes. These are the rapid development modern information technology [5]. But electronic technology is still like before. Although the development of these years has not fallen, the current electronic technology in the current change will often appear some frequency fluctuations to interfere. And in high-precision electronic products, because small components can not be disturbed by frequency, otherwise they will be damaged and scrapped, so this is a huge test for modern electrical and electronic technology. So we need to study it [6].Therefore, this paper thinks of the reform of electrical and electronic technology and the intelligent reform of computer technology to increase its reform progress and adaptability. But these are very difficult, because there are many factors, we can not guarantee complete success, it takes many attempts to get the data we need [7].

Because it has a lot of interference, such as external magnetic field interference and internal eddy current interference [8]. Because the electromagnetic field is not separated, there will be magnetism in the place where there is electricity, there will be electricity in the place where there is magnetism, and the magnetic field will often produce magnetic field around the periphery when the electricity is transported, and these magnetic fields will produce electricity to hinder the work of the original current. As a result, the original set of work efficiency and our actual work efficiency errors, these are very common, 
but to eliminate it is very difficult to [9]. Because this is the result of natural laws, we can not violate the laws of nature, so we can only reduce the error in a very small range to produce controllable changes in the experiment. In this way, we can better carry out the research and development of electrical and electronic technology. So we need to intelligently deploy it, so that it can better adapt itself to achieve the purpose we want to achieve. According to the current technology, the use of artificial intelligence technology is the best choice [10].

\section{Adaptive parameter DBSCAN clustering algorithm}

Generally, in clustering analysis, the mean square integral error function (MISE) is generally selected to optimize the bandwidth. They are defined as follows:

$$
\operatorname{MISE}(h)=E \int(\hat{f}(x)-f(x))^{2} d x
$$

Under the weak assumption:

$$
\begin{aligned}
& \operatorname{MISE}(h)=\operatorname{AMISE}(h)+o\left(\frac{1}{(n h)}+h^{4}\right) \\
& \operatorname{AMISE}(h)=\frac{R(K)}{n h}+\frac{1}{4} m_{2}(K)^{2} h^{4} R\left(f^{\prime \prime}\right)
\end{aligned}
$$

Among them:

$$
\begin{aligned}
R(K) & =\int K(x)^{2} d x \\
m_{2}(K) & =\int x^{2} K(x) d x
\end{aligned}
$$

Minimizing MISE (h) is equivalent to minimizing AMISE (h), finding the partial derivative and making the derivative equal to 0 :

$$
\begin{aligned}
& \frac{\partial}{\partial h} A M I S E(h)=-\frac{R(k)}{n h^{2}}+m_{2}(K)^{2} h^{3} R\left(f^{\prime \prime}\right)=0 \\
& h_{A M I S E}=\frac{R(K)^{\frac{1}{5}}}{m_{2}(K)^{\frac{2}{5}} R\left(f^{\prime \prime}\right)^{\frac{1}{5}} n^{\frac{1}{5}}}
\end{aligned}
$$

Among them $\mathrm{m} 、 \mathrm{R}$ according to the kernel function.

\section{Experimental Establishment}

\subsection{Selection and treatment of experiments}

Because this experiment is mainly related to electrical engineering and electrical and electronic technology, we need to borrow an electrical laboratory to complete the experiment. Therefore, first we borrowed an electrical and electronic technology laboratory from the school, and then we set the program through the system for automatic control and set the data to complete the experiment. We look at the data in the experiment to see if we can agree on the error within a small error range, and then approximate the error to a minimum range through multiple experiments. Then by finding out the reasons to analyze the success of the experiment, record all the information, complete the experiment.

\subsection{Processing of experimental data}

Because there are a lot of data to be completed, the amount of data to be measured is relatively large, so we get two thousand pairs of data through 20 groups of repeated experiments. Then ten groups of the best experimental data are selected to approximate the optimal data obtained under artificial intelligence technology, and then the experimental results are obtained by comparing the prediction design.

\section{Evaluation results}

\subsection{Experimental results}

Table 1.Comparative analysis of experimental data

\begin{tabular}{|l|l|l|l|l|}
\hline & $\begin{array}{l}\text { Minimum } \\
\text { constraint value }\end{array}$ & $\begin{array}{l}\text { Maximum } \\
\text { fluctuation } \\
\text { difference }\end{array}$ & $\begin{array}{l}\text { Minimum } \\
\text { fluctuation } \\
\text { difference }\end{array}$ & $\begin{array}{l}\text { Stable } \\
\text { volatility }\end{array}$ \\
\hline Experiment 1 & 0.012 & 0.822 & 0.102 & 0.420 \\
\hline Experiment 2 & 0.011 & 0.762 & 0.086 & 0.391 \\
\hline Experiment 3 & 0.012 & 0.832 & 0.114 & 0.411 \\
\hline Experiment 4 & 0.007 & 0.841 & 0.104 & 0.422 \\
\hline Experiment 5 & 0.013 & 0.811 & 0.092 & 0.422 \\
\hline
\end{tabular}

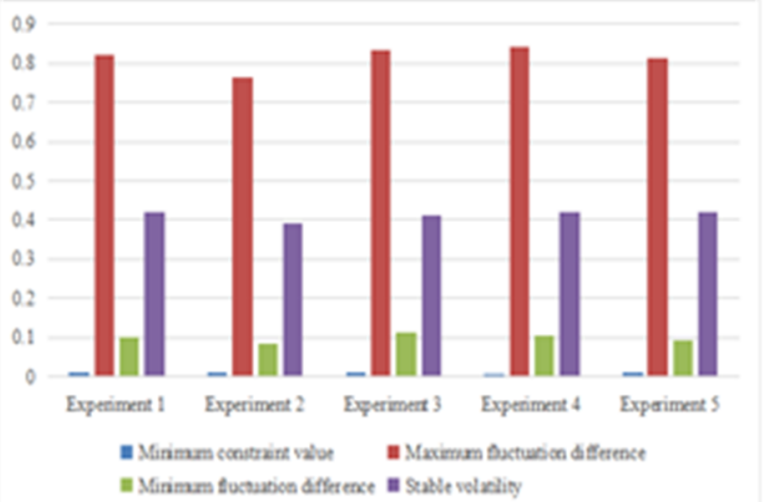

Figure 1.Comparative analysis of experimental data

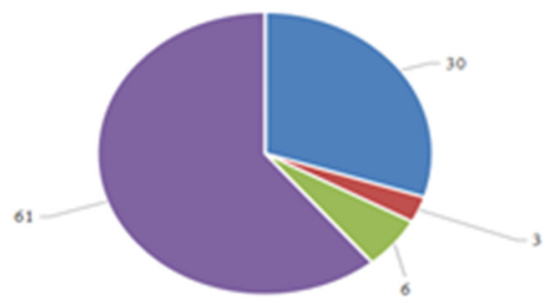

Figure 2 .Abnormal Data Analysis

According to the results of Table 1 and Figure 1, Figure 2, we can know that the experiment is quite good, so our next step is to reduce the error and get better data representation results.

\subsection{Electrical engineering}

It is because of the great progress of electronic technology that the modern information age based on computer network is promoted. And changed the habits of the whole society, from the original artificial into the modern intelligent era. There are many courses in electrical engineering, but the main subjects are circuit principle, signal and system control, electronic technology and so on. At present, electrical engineering is developing very fast, because electrical engineering and information technology complement each other. Although electricity first promoted the development of information technology, the 
development of information technology also promoted the development and innovation of electrical engineering. And the development of information technology provides a lot of tool basis for electrical engineering. For example, the communication between electrical engineering and physical science produces diodes, transistors, integrated circuits and so on. Then the information technology makes the intelligent decision for the control of electrical engineering because of the extremely high efficiency and the extremely high control rate, so that the efficiency is optimized. Because electric power is the foundation of modern life, the application of electric power in the world is also being explored and optimized. Therefore, in the future, the subject related to electricity will still be developed, and electrical engineering will still become one of the mainstream disciplines in society. Electrical engineering is a major first-level discipline, which is later divided into many categories, such as communications and networks, signal processing, electronics and integrated circuits, computer science and engineering system control, photonics and optics, power technology, electromagnetism, Microstructures, materials and devices, etc. These are the future development direction of electrical engineering, compared with other majors, these directions are generally related to electrical engineering, can get greater progress in these directions and continue to improve themselves, expand their knowledge.

Electrical automation is generally used in industrial control. Because modern industry has been slowly replaced by machines from the original manual production, now mainly by people to control the system to complete the production. The requirement of automatic operation of machine mainly comes from electrical automation. Electrical automation is the process of using relays and other components to realize the control, and then through other auxiliary devices to control the internal operation to achieve the purpose of electrical automation.

The most important and basic thing in electrical engineering is power supply, because any power supply equipment can not work without power supply. The power supply is also divided into many kinds, but all of our tools are pursuing stable and superior power supply, so it can be said that the development of power supply indirectly promotes the development of the whole electrical industry. Power supply from many years ago DC to later AC, and $100000 \mathrm{v}$ into $110 \mathrm{v}, 220 \mathrm{v}, 380 \mathrm{v}$ and other different output voltages, the power supply after many years of reform has slowly become more and more stable. But for some highprecision products, they have a high demand for power. The industrial power supply we use generally has some errors, so it is not applicable. so we need frequency stable voltage in demanding electronics. The AC conversion will generally appear deviation, so in high-precision electronic products generally use DC power supply. With the development of computer, the grade is improving and the function is becoming stronger and stronger. The power revolution will further penetrate into various industries and affect the material life of all mankind.

\subsection{Electrical and electronic technology}

The modern electrical and electronic foundation can generally be traced back to the birth of the world's first electronic and digital computer in 1946. Computers are generally divided into four stages of development, From 1946 1957, 1958 1964, 1965 1971 and 1972 to present. The difference between them is mainly the construction of basic components. The components of the first generation are electronic tubes, the second generation of building blocks is transistors, the third generation is small, Medium scale integrated circuits, the fourth generation of building components for large-scale and very large-scale integrated circuits. For computers, Feng Neumann, the father of computer, put forward a general computer design scheme. He made three requests, First, the computer has five basic functions, the operator, controller, memory, input equipment and output equipment. Second, computers generally use binary numbers to express instructions and data. Third, all programs and data are stored in memory, Then the computer automatically executes the woven program. And with the development of modern science and technology, the future direction of computer development should be divided into two directions: giant and miniaturization. Supercomputers, Like Tianhe 2, Microcomputers generally refer to laptops and home computers now in general use. Microcomputers were invented after the fourth generation of computers, because it is the product of large-scale integrated circuits. And with all these years of development, Great progress has also been made, From the first four digits to 16 to 32, And finally, the 64 bits we're using, And its integration from the original 2300 to the present 42 million. These are the great improvement of computer technology caused by the development of the times. And in the future, Computers will be more convenient to share data than they are now. And with the development of artificial intelligence technology, in the future computers may also be intelligent, Simulate the human mind, And has the human logical reasoning and the study ability.

\section{Conclusion}

To sum up, after consulting the relevant data and carrying out experiments, we find that it still needs a long way to go to intelligentize the electrical and electronic technology at this stage. At present, we can only roughly set the program to let the computer follow the program to automate the control of electronics. However, because there are many interference factors, the experimental results are not very ideal, and some data comparison is needed to exclude the required data. Therefore, after a large number of experiments, we can draw a general conclusion that the current electrical and electronic technology. Can not be fully intelligent, can only rely on the set of procedures for semi-intelligent control. Perhaps when artificial intelligence technology is further improved in the future, electrical and electronic technology can be fully intelligent to solve the problems we face. 


\section{References}

1. A P A I, B X Z, Jacob Vanhouten, M C, et al. Artificial Intelligence for Diagnosis of Acute Coronary Syndromes: A Meta-analysis of MachineLearning Approaches[J]. Canadian Journal of Cardiology, 2020, 36( 4):577-583.

2. Pitt C S, Bal A S, Plangger K. New approaches to psychographic consumer segmentation: Exploring fine art collectors using artificial intelligence, automated text analysis and correspondence analysis[J]. European Journal of Marketing, 2020, 54(2):305-326.

3. Wang Y, Liu Z, Qu A, et al. Study of tacrine derivatives for acetylcholinesterase inhibitors based on artificial intelligence[J]. LATIN AMERICAN JOURNAL OF PHARMACY, 2020, 39(6):1159-1170.

4. Lager I E, Vandenbosch G A E, Stumpf M . Electromagnetism in the Electrical Engineering Classroom: Dominant trends in teaching classical electromagnetic field theory and innovation vectors[J]. IEEE Antennas and Propagation Magazine, 2020, 62(2):14-23.

5. Li R, Xi-xi Sun, Zheng T, et al. Defect engineering electrical properties of lead-free potassium sodium niobate-based ceramics[J]. Journal of the American Ceramic Society, 2020, 103(1):444-453.

6. Praveenasri P . Water Efficiency Management Strategies in Electric Engineering Generation Industries: an Epilogue PeriniPraveenaSri[J]. International Journal of Engineering and Advanced Technology, 2020, 9(1):840-842.

7. Haruna, R., Kamin, Y.B., Buntat, Y.B., Shuaibu, H., Cledumas, A.M. Functional Approaches for Applying Workbased Learning into Electrical and Electronic

8. Technology Education in Nigeria[J]. International Journal of Engineering and Advanced Technology, 2019, 8(5):831-839.

9. Khan M I , Rasheed I, Naeem M A, et al. Structural, electrical and mechanical behavior evaluation of palladium doped diamond like carbon thin films[J]. Journal of Materials Research and Technology, 2020, 9(4):8289-8295.

10. Huang $\mathrm{H} \mathrm{C}$, Ho Y C . Applying Intelligent Technology in Gender Competence Training for Nurses[J]. Hu li za zhi The journal of nursing, 2019, 66(2):29-35.

11. Niu W, Zhao P . Research on Training Model of Innovative Practical Talents Based on Intelligent Technology[J]. World Scientific Research Journal, 2019, 5(11):44-47. 